EUROPHYSICS LETTERS

Europhys. Lett., 73 (2), pp. 183-189 (2006)

\title{
New Green-Kubo formulas for transport coefficients in hard sphere-, Langevin fluids and the likes
}

\author{
M.H. ERnst ${ }^{1,2}$ AND R. BRITO ${ }^{2}$ \\ ${ }^{1}$ Institute Theoretical Physics, Universiteit Utrecht, 3508 TD Utrecht, The Netherlands \\ ${ }^{2}$ Dpt. Física Aplicada I and GISC, Universidad Complutense, 28040 Madrid, Spain
}

(received ; accepted )

PACS. 05.40.-a - Fluctuation phenomena, random processes, noise and Brownian motion. PACS. 05.20.Dd- Kinetic theory.

\begin{abstract}
. -
We present generalized Green-Kubo expressions for thermal transport coefficients $\mu$ in nonconservative fluid-type systems, of the generic form, $\mu=\mu_{\infty}+\int_{0}^{\infty} d t V^{-1}\left\langle I_{\epsilon} \exp (t \mathcal{L}) I\right\rangle_{0}$ where $\exp (t \mathcal{L})$ is a pseudo-streaming operator. It consists of a sum of an instantaneous transport coefficient $\mu_{\infty}$, and a time integral over a time correlation function in a state of thermal equilibrium between a current $I$ and its conjugate current $I_{\epsilon}$. This formula with $\mu_{\infty} \neq 0$ and $I_{\epsilon} \neq I$ covers vastly different systems, such as strongly repulsive elastic interactions in hard sphere fluids, weakly interacting Langevin fluids with dissipative and stochastic interactions satisfying detailed balance conditions, and "the likes", defined in the text. For conservative systems the results reduce to the standard formulas.
\end{abstract}

The Green-Kubo formulas for thermal transport coefficients in simple classical fluids with smooth conservative interactions are widely used, and generally accepted $[1,2,3]$ as exact expressions for general densities, as long as the deviations from equilibrium and the gradients are small, and the transport coefficients exist. This standard expression is given in terms of an equilibrium time correlation function between $N$-particle currents $I$ and $I_{\epsilon}$, i.e.

$$
\mu=\int_{0}^{\infty} d t \lim _{V \rightarrow \infty} \frac{1}{V}\langle I(0) I(t)\rangle_{0},
$$

where $\mu$ denotes a typical transport coefficient, $\langle\ldots\rangle_{0}$ is an average over a thermal equilibrium ensemble at temperature $T=1 / k_{B} \beta$, with a distribution function $\rho_{0} \sim \exp [-\beta H]$, and $\lim _{V \rightarrow \infty}$ denotes the thermodynamic limit. In the sequel this limit is understood, but not explicitly written. For convenience, time is taken to be continuous. The time evolution of a dynamical variable, $I(t)=e^{t \mathcal{L}} I(0)$, can be described by a streaming operator $e^{t \mathcal{L}}$, which generates a time evolution that is invariant under the time reversal transformation. Consequently, the Liouville operator satisfies, $\mathcal{L}^{\epsilon}=-\mathcal{L}$, where $\mathcal{L}^{\epsilon}$ denotes the time reversal transform of $\mathcal{L}$. As $\mathcal{L}$ and the currents $I$ contain in general interparticle forces, the standard Green-Kubo formula (1) is ill-defined if the interaction potentials are not sufficiently smooth,

Typeset using EURO-TEX 
and it does neither apply to hard sphere or hard core interaction potentials (non-conservative impulsive forces), nor to dissipative and stochastic forces, which are not derivable from an interaction potential.

Problems addressed: The goal of this letter is to present generalized Green-Kubo expressions, without any restrictions to dilute systems, for a large class of complex (non-conservative) fluids, i.e. $N$-particle systems, that do approach a state of thermal equilibrium, but whose equations of motion have ill-defined forces, or/and lack time reversal invariance. The forces may be impulsive, dissipative, stochastic, and possibly include conservative forces as well. The generic form of this generalized Green-Kubo formula is,

$$
\mu=\mu_{\infty}+V^{-1} \int_{0}^{\infty} d t\left\langle I_{\epsilon} e^{t \mathcal{L}} I\right\rangle_{0},
$$

where it must be possible to represent the time evolution through a pseudo-streaming operator $\exp (t \mathcal{L})$, which is well-defined for all points in the relevant phase space, and generates the proper trajectories of the dynamical variables when used inside statistical averages $\langle\cdots\rangle_{0}$. The prefix pseudo refers to the fact that the representation $\exp [t \mathcal{L}]$ is only valid inside averages. An assessment of the type of systems to which this Green-Kubo formula is also applicable, called "the likes", and an outline of the derivation and implications are the main goals of the present article. The discussion covers such vastly different systems as microscopic fluid models with strongly repulsive elastic interactions (hard spheres, square well potentials) for which the interparticle pseudo-forces and the pseudo-Liouville operators in Eq.(2) are well-defined, as well as mesoscopic weakly interacting Langevin fluids with dissipative and stochastic forces.

There seem to exist in the literature only three cases where explicit Green-Kubo formulas of the generalized form (2) have been derived for transport coefficients in complex fluids, each referring to a very different system and to a very different transport coefficient, obtained by very different approaches. The first case in Ref.[4] refers to Brownian dynamics. Here the friction coefficient of a Brownian particle in a fluid, both modeled by hard spheres, is derived with the help of a delicate analysis, using an asymptotic expansion in the small ratio $m / M$ of the hard sphere mass over the mass of the Brownian particle. In this limit the interactions between the heavy particle and the liquid particles becomes weak, and the kinetic equation for the distribution function of the heavy particle reduces to a Fokker Planck equation. Here the friction coefficient is expressed as a generalized Green-Kubo formula (2), and contains a time correlation function between a pseudo-interparticle force and its conjugate. A possible extension of this method to complex fluids is not obvious.

A second case, also concerning hard spheres, can be found in Ref. [5] for the shear viscosity of an elastic hard sphere fluid, derived by a transformation of the corresponding EinsteinHelfand formula, or by a limiting procedure applied to the standard Green-Kubo formulas for soft spheres with a repulsive interaction potential $V(r) \sim 1 / r^{n}$ with $n \rightarrow \infty$. The limiting procedure cannot be extended to complex fluids, but the first route via an Einstein-Helfand formula is possible, following Ref. [5].

A third case concerns a Langevin fluid [6], where standard linear response theory [1] is used to obtain the long time diffusion coefficient of a colloidal suspension in the generic form (2). This is done by modeling the short time diffusion coefficient by a phenomenological relaxation constant, used as input in the Langevin equation. The extension of that method for a weakly interacting Langevin fluid to strongly interacting hard sphere systems does not seem feasible either.

The question of interest in this article is then: do these scattered results have something in common that makes the structure (2) generic for a larger class of physical systems? The answer to this question will be given below. 
Properties of $\mathcal{L}$ : Suppose that $\mathcal{L}$ has been constructed. As we are studying equilibrium time correlation functions, we have to impose the condition of stationarity,

$$
\langle A(0) B(t)\rangle_{0}=\langle A(-t) B(0)\rangle_{0} .
$$

Here the time evolution of $A(-t) \equiv \exp \left(t \mathcal{L}^{\epsilon}\right) A(0)$ (where $t>0$ ) is generated by the backward or time reversed streaming operator. Defining the transpose $\widetilde{\mathcal{L}}$ through the relation, $\int d \Gamma A e^{t \mathcal{L}} B=$ $\int d \Gamma B e^{t \widetilde{\mathcal{L}}} A$, we find by comparing integrands in Eq. (3) the necessary condition on $\mathcal{L}$ in the form of a commutation relation, i.e.

$$
\rho_{0} \mathcal{L}=\widetilde{\mathcal{L}}^{\epsilon} \rho_{0} \quad \text { or } \quad \overline{\mathcal{L}}=\widetilde{\mathcal{L}}^{\epsilon},
$$

where $\overline{\mathcal{L}}$ is defined through $\rho_{0} \mathcal{L} \equiv \overline{\mathcal{L}} \rho_{0}$. The above ingredients are sufficient to derive Green-Kubo type formulas for the Navier-Stokes transport coefficients using linear response theory. The class of "the likes" consists of those microscopic or mesoscopic models that have: (i) a pseudo-streaming operator of the form $\exp [t \mathcal{L}]$, (ii) a stationary distribution given by the thermal $\rho_{0}$, where (iii) $\rho_{0}$ and $\mathcal{L}$ satisfy the commutation relation (4). Note that for conservative interactions, where $\widetilde{\mathcal{L}}=-\mathcal{L}=\mathcal{L}^{\epsilon}$, the condition (4) is trivially satisfied as $\overline{\mathcal{L}}=\widetilde{\mathcal{L}}^{\epsilon}=\mathcal{L}$, and $\mathcal{L}$ and $\rho_{0}$ commute.

There exist two important simple realizations of the previous scenario. The first and most important class are the fully microscopic models of hard sphere fluids, which are prototypical for the strongly repulsive hard core interactions of classical fluids. The other class are mesoscopic Langevin fluids with weak, dissipative and stochastic forces, where the interactions are modeled by phenomenological input parameters.

For hard spheres the standard Liouville operator for conservative systems is ill-defined because of the impulsive interaction forces. For this case the construction of pseudo-Liouville operators $\mathcal{L}$ in terms of binary collision operators is far from trivial [7]. The resulting expressions for $\mathcal{L}$ in terms of binary collision operators satisfy the commutation relation (4), as shown in [7]. This pseudo-Liouville operator is not odd in the velocities. So $\mathcal{L}^{\epsilon} \neq-\mathcal{L}$, and consequently not all trajectories generated by $\exp (t \mathcal{L})$ are time reversal invariant.

For Langevin fluids the construction of the pseudo-Liouville operator $\mathcal{L}$ is rather trivial, as this operator can be simply obtained from the corresponding Fokker-Planck equation, $\partial_{t} \rho=\widetilde{\mathcal{L}} \rho$, for the $N$-particle probability distribution $\rho(\Gamma, t)[8]$. As this equation satisfies the detailed balance condition, the stationary solution of $\partial_{t} \rho_{0}=\widetilde{\mathcal{L}} \rho_{0}=0$ is the thermal distribution $\rho_{0}$. Also here it is sufficient to define pseudo-streaming operators $e^{t \mathcal{L}}$ for the time evolution of dynamical variables only inside averages. This may be done through the relation,

$$
\langle A(t)\rangle=\int d \Gamma A e^{t \widetilde{\mathcal{L}}} \rho(\Gamma, 0)=\int d \Gamma \rho(\Gamma, 0) e^{t \mathcal{L}} A=\left\langle e^{t \mathcal{L}} A\right\rangle .
$$

Here the pseudo-Liouville operator $\mathcal{L}$ is the transpose of the Fokker-Planck operator. In this formulation $\mathcal{L}$ is not the infinitesimal generator of the Langevin equations, but an effective operator, that acts inside averages, in which the rapid fluctuations of the random forces have been averaged out. Because of the presence of dissipative forces, the Fokker-Planck equation is not invariant under the transformation of time reversal. Consequently, $\mathcal{L}^{\epsilon} \neq-\mathcal{L}$. For Fokker-Planck equations, satisfying the detailed balance criteria, the operator $\mathcal{L}$ satisfies the commutation relation (4) (see [8]).

Linear response theory: Having discussed the properties of the pseudo-Liouville operator, one can apply linear response theory for calculating thermal transport coefficients in fluids $[1,3]$. Consider, for simplicity, a system with a single conserved density $e(\mathbf{r})$. Extensions to more conservation laws are straightforward. Let $e_{\mathbf{k}}$ be the Fourier mode of the conserved 
density, $e_{\mathbf{k}}=\sum_{i} \delta \epsilon_{i} \exp \left[-i \mathbf{k} \cdot \mathbf{r}_{i}\right]$ with fluctuation $\delta \epsilon_{i}=\epsilon-\langle\epsilon\rangle_{0}$. The microscopic equation of motion for the Laplace transform $e_{\mathbf{k} z}$ of $e_{\mathbf{k}}(t)$ is $(z-\mathcal{L}) e_{\mathbf{k} z}=e_{\mathbf{k}}$. In the long wave length limit $(k \rightarrow 0)$ the hydrodynamic propagator satisfies the relation,

$$
G(\mathbf{k}, z) \equiv\left\langle e_{\mathbf{k}} \mid e_{\mathbf{k} z}\right\rangle \simeq\left\langle e_{\mathbf{0}} \mid e_{\mathbf{0}}\right\rangle /\left[z+k^{2} D\right]
$$

where $D=\mu /\left\langle e_{\mathbf{0}} \mid e_{\mathbf{0}}\right\rangle$ is the diffusivity, and $\mu$ the kinetic coefficient. The standard projection operator method [1] enables us to derive in a few lines an exact expression for $D$ or $\mu$. To do so we introduce the projection operator $\mathcal{P}=1-\mathcal{P}_{\perp}$ acting on a dynamic variable $b_{\mathbf{k}}$ as $\mathcal{P} b_{\mathbf{k}}=$ $e_{\mathbf{k}}\left\langle e_{\mathbf{k}} \mid b_{\mathbf{k}}\right\rangle /\left\langle e_{\mathbf{k}} \mid e_{\mathbf{k}}\right\rangle$. The inner product is defined as a thermal average, $\left\langle a_{\mathbf{k}} \mid b_{\mathbf{k}}\right\rangle=V^{-1}\left\langle a_{\mathbf{k}}^{*} b_{\mathbf{k}}\right\rangle_{0}$. Application of this method yields,

$$
\left[z-\mathcal{P} \mathcal{L P}-\mathcal{P} \mathcal{L} \mathcal{P}_{\perp}\left(z-\mathcal{P}_{\perp} \mathcal{L} \mathcal{P}_{\perp}\right)^{-1} \mathcal{P}_{\perp} \mathcal{L P}\right] \mathcal{P} e_{\mathbf{k} z}=e_{\mathbf{k}}
$$

Writing this in component form, and comparing the result with (6) yields for $D$,

$$
D=-\mathcal{L} i m \Re\left[\left\langle e_{\mathbf{k}} \mid \mathcal{L} e_{\mathbf{k}}\right\rangle+\left\langle\mathcal{L}^{\epsilon} e_{\mathbf{k}} \mid \mathcal{P}_{\perp} \hat{\mathcal{G}}_{z} \mathcal{P}_{\perp} \mathcal{L} e_{\mathbf{k}}\right\rangle\right] /\left[k^{2}\left\langle e_{\mathbf{k}} \mid e_{\mathbf{k}}\right\rangle\right],
$$

where $\mathcal{L}$ im represents the double $\operatorname{limit}, \lim _{z \rightarrow 0} \lim _{k \rightarrow 0}, \Re$ denotes the real part, and $\hat{\mathcal{G}}_{z}$ reduces in the small- $k$ limit to the projected resolvent, $\mathcal{P}_{\perp}(z-\mathcal{L})^{-1} \mathcal{P}_{\perp}$. To obtain the second term we have used the relation $\left\langle e_{\mathbf{k}} \mid \mathcal{L} \mathcal{P}_{\perp} \cdots\right\rangle=\left\langle\mathcal{L}^{\epsilon} e_{\mathbf{k}} \mid \mathcal{P}_{\perp} \cdots\right\rangle$, based on the commutation relation (4). This term can be expressed as a current-current correlation function.

Consider the so called Euler matrix $\left\langle e_{\mathbf{k}} \mid \mathcal{L} e_{\mathbf{k}}\right\rangle=-i k\left\langle e_{\mathbf{k}} \mid j_{\mathbf{k}}\right\rangle$. Its imaginary part would yield the Euler equations for a fluid with the standard conservation laws. As conserved densities have in general a definite parity in the velocities (mass, momentum, energy), the Euler matrix element would vanish for conservative systems, where $\mathcal{L}^{\epsilon}=-\mathcal{L}$. However, for systems whose pseudo-streaming operator lacks time reversal invariance, $\mathcal{L}^{\epsilon} \neq-\mathcal{L}$, and the matrix element has a non-vanishing real part for small $k$ of $\mathcal{O}\left(k^{2}\right)$, i.e.

$$
\mu_{\infty}=\left\langle e_{\mathbf{0}} \mid e_{\mathbf{0}}\right\rangle D_{\infty}=-\lim _{k \rightarrow \infty} k^{-2} \Re\left\langle e_{\mathbf{k}} \mid \mathcal{L} e_{\mathbf{k}}\right\rangle .
$$

Next consider the second term in Eq. (8). Here the total microscopic flux, $J=\lim _{k \rightarrow 0} j_{\mathbf{k}}$, is related to the Fourier mode $e_{\mathbf{k}}$ of the conserved density through the local conservation law, $\partial_{t} e_{\mathbf{k}}=\mathcal{L} e_{\mathbf{k}}=-i k j_{\mathbf{k}}$. Similarly, we define the conjugate current, $J_{\epsilon}=\lim _{k \rightarrow 0} j_{\epsilon \mathbf{k}}^{*}$ through $\mathcal{L}^{\epsilon} a_{\mathbf{k}}^{*}=-i k j_{\epsilon \mathbf{k}}^{*}$. Inserting these definitions into Eq. (8) yields finally for the kinetic coefficient $\mu$ the generalized Green-Kubo formula (2), where $I$ and $I_{\epsilon}$ are subtracted currents, defined as $I=\mathcal{P}_{\perp} J$. This completes the formal derivation of Eq. (2), and it remains to work out the explicit expressions for $I, I_{\epsilon}$ and $\mu_{\infty}$. In the remaining part of this letter we present some applications to a heat conducting random solid, an isothermal Langevin fluid, and a hard sphere fluid.

Heat conducting random solid: A realization of a system in the previous section is a quenched Langevin fluid with heat conduction. It consists of $N$ point particles, quenched in a random configuration $X=\left\{\mathbf{r}_{i} \mid i=1, \cdots, N\right\}$. Each particle is characterized by its internal energy $\epsilon_{i}$, collectively denoted by $\mathbf{e}=\left\{\epsilon_{i}(t) \mid i=1, \cdots, N\right\}$, which are the only dynamical variables in the model. The internal energy corresponds to many internal degrees of freedom, described by a density of states $\sim \epsilon^{\alpha}$, where $\alpha$ is proportional to the number of internal degrees of freedom $(\alpha \gg 1)$. Energy is exchanged between the particles through dissipative and stochastic forces of finite range, which obey detailed balance criteria. The total energy, $E=\sum_{i} \epsilon_{i}(t)$ is conserved, and the $N$-particle system approaches a state of thermal equilibrium. The system supports a local heat current, driven by gradients in the local energy density or temperature field. So, at the macroscopic level Fourier's law of heat conduction applies, at least if the density of particles 
is above a critical percolation threshold. Our goal is to derive a Green-Kubo expression for its heat conductivity.

The Langevin equations for the time evolution of the dynamical variables $\epsilon_{i}(t)$ and the corresponding Fokker-Planck equation for the $N$-particle probability density $\rho(\mathbf{e}, t \mid X)$ in a fixed configuration $X$ of $N$ point particles have been derived to dominant order $\mathcal{O}(1 / \alpha)$ in Ref. $[9,10]$. We only quote the adjoint Fokker-Planck operator $\mathcal{L}$ which reads,

$$
\mathcal{L}=\sum_{i<j} \lambda_{i j}\left[\left(\epsilon_{j}-\epsilon_{i}\right)+\alpha^{-1} \epsilon_{i} \epsilon_{j} \partial_{i j}\right] \partial_{i j}=\mathcal{L}^{\epsilon} .
$$

Here $\partial_{i j}=\partial / \partial \epsilon_{i}-\partial / \partial \epsilon_{j}$, and $\lambda_{i j}=\lambda_{0} w\left(r_{i j}\right)$ with a positive range function $w\left(r_{i j}\right)$ vanishing for $r_{i j} \geq r_{c}$, and $\gamma_{0}$ is a phenomenological model parameter. The equation above shows that $\mathcal{L}^{\epsilon}=\mathcal{L}$ because $\epsilon_{i}$ does not change sign under a time reversal transformation. The microscopic energy fluxes for small $k$ are obtained as [10],

$$
\begin{aligned}
\mathcal{L} e_{\mathbf{k}} & \simeq-i \mathbf{k} \cdot \sum_{i<j} \lambda_{i j}\left(\epsilon_{j}-\epsilon_{i}\right) \mathbf{r}_{i j} \equiv-i \mathbf{k} \cdot \mathbf{Q} \\
\mathcal{L}^{\epsilon} e_{\mathbf{k}}^{*} & \simeq-i \mathbf{k} \cdot \mathbf{Q}_{\epsilon}=\mathcal{L} e_{\mathbf{k}}^{*}=+i \mathbf{k} \cdot \mathbf{Q},
\end{aligned}
$$

and there are no subtracted parts. Note the relation $\mathbf{Q}_{\epsilon}=-\mathbf{Q}$. The Green-Kubo formula for heat conductivity in the random solid is then $\lambda=\lambda_{\infty}+\lambda^{d d}$, with

$$
\lambda^{d d}=(\beta / d T V) \int_{0}^{\infty} d t\left\langle\mathbf{Q}_{\epsilon} \cdot e^{t \mathcal{L}} \mathbf{Q}\right\rangle_{0}=-(\beta / d T V) \int_{0}^{\infty} d t\left\langle\mathbf{Q} \cdot e^{t \mathcal{L}} \mathbf{Q}\right\rangle_{0} .
$$

The Green-Kubo expression (12) has the generic form for systems with dynamics lacking time reversal invariance. As this formula can also be derived from an Einstein-Helfand formula, $\lambda$ is necessarily positive. Because $\mathcal{L}^{\epsilon} \neq-\mathcal{L}$, necessarily $\lambda_{\infty} \neq 0$. The instantaneous heat conductivity is equal to the mean field approximation to the heat conductivity, calculated in Refs. $[9,10]$, i.e. $\lambda_{\infty}=\left(\lambda_{0} n^{2} C_{v} / 2 d\right)\left[w R^{2}\right]$ where $\left[w R^{2}\right]=\int d \mathbf{R} w(\mathbf{R}) R^{2}$ and $C_{v}$ is the specific heat per particle. In fact, the remaining time integral in (12) gives at large densities only a negligible contribution, but at the percolation threshold both terms cancel [10]. If the standard Green-Kubo formula (1) would have applied, then $\lambda_{\infty}=0$, and the sign in front of the second time integral would have been a plus sign. So, the resulting expression would have been quite different from Eq. (12).

Isothermal DPD fluid: Next we will consider the so called isothermal DPD (dissipative particle dynamics) fluid, introduced in Ref.[11], and studied analytically [12, 13] and by means of computer simulations [14]. It is a mesoscopic version of a classical fluid where the fast microscopic length and time scales are averaged out, and DPD particles, described by their position $\mathbf{r}_{i}$ and velocity $\mathbf{v}_{i}$, can be thought of as soft lumps of fluid. Their equations of motion, $d \mathbf{r}_{i} / d t=\mathbf{v}_{i}$ and $m d \mathbf{v}_{i} / d t=\sum_{j}\left(\mathbf{F}_{i j}^{c}+\mathbf{F}_{i j}^{d}+\mathbf{F}_{i j}^{r}\right)$, contain conservative forces, $\mathbf{F}_{i j}^{c}=-\partial V\left(r_{i j}\right) / \partial \mathbf{r}_{i j}$, as well as dissipative $(d)$ and random $(r)$ forces, where $\mathbf{F}^{d}$ is proportional to a phenomenological relaxation parameter $\gamma_{0}$. Explicit expressions for $\mathbf{F}^{d}$ and $\mathbf{F}^{r}$ can be found in Refs. [12, 13]. All forces have a finite mesoscopic range $r_{c}$. The corresponding Fokker-Planck equation satisfies the detailed balance condition. The pseudo-Liouville operator, $\mathcal{L}=\mathcal{L}_{c}+\mathcal{L}_{d}$, is obtained as the transpose of the Fokker-Planck operator. Its conservative part $\mathcal{L}_{c}$ includes the inertial term and the conservative forces (where $\mathcal{L}_{c}^{\epsilon}=-\mathcal{L}_{c}$ ). Its dissipative part $\mathcal{L}_{d}$ contains the combined action of the dissipative and stochastic forces (where $\mathcal{L}_{d}^{\epsilon}=\mathcal{L}_{d}$ ). Consequently the instantaneous transport coefficient depends solely on the dissipative force. In the DPD fluid total mass and momentum are conserved, but not the total energy. So mass density $n_{\mathbf{k}}$ and momentum density $\mathbf{g}_{\mathbf{k}}$ are the only slow Fourier modes. Therefore, the fluid exhibits shear and bulk viscosity. The transport coefficients are given by the matrix generalization of Eq.(8) (see [3]). Here we restrict ourselves to the shear viscosity $\eta$. It is 
given by an expression similar to (8) with $e_{\mathbf{k}}$ replaced by the transverse momentum density $g_{\mathbf{k} \perp}=\sum_{i} m v_{i y} \exp \left[-i k r_{i x}\right]$ where $\mathbf{k}$ is taken parallel to the $x$-axis for convenience, and it is straight forward to obtain,

$$
\begin{aligned}
\eta & =\eta_{\infty}+(\beta / V) \int_{0}^{\infty} d t\left\langle\left(J_{x y}^{c}-J_{x y}^{d}\right) e^{t \mathcal{L}}\left(J_{x y}^{c}+J_{x y}^{d}\right)\right\rangle_{0} \\
& =\eta_{\infty}+\eta^{c c}+\eta^{c d}+\eta^{d c}+\eta^{d d}
\end{aligned}
$$

For small $k$ the relation $\mathcal{L} g_{\mathbf{k} \perp} \simeq-i k J_{x y}$ has been used, where the total momentum current $J_{x y}=J_{x y}^{c}+J_{x y}^{d}$, and $J_{\epsilon x y}=J_{x y}^{c}-J_{x y}^{d}$. Here $J_{x y}^{c}$ is the momentum current in the standard Green-Kubo formula for conservative forces, given here by $\eta^{c c}$, and $J_{x y}^{d}=-J_{\epsilon, x y}^{d}=$ $\sum_{i<j} r_{i j, x} F_{i j, y}^{d}$ is the dissipative current. The instantaneous viscosity $\eta_{\infty}$, which is proportional to $k^{-2}\left\langle g_{\mathbf{k} \perp} \mid \mathcal{L} g_{\mathbf{k} \perp}\right\rangle$, is equal to the quantity $\eta_{D}=m n^{2} \gamma_{0}\left[g_{0} w R^{2}\right] / 2 d(d+2)$, where $g_{0}(r)$ is the radial distribution function in thermal equilibrium, calculated in Ref.[11, 13] as the mean field approximation to the viscosity. Also note that the commutation relation (4) implies that $\eta^{c d}=\eta^{d c}$, which contributions are in general non-vanishing. If the standard Green-Kubo formula would apply, then $\eta_{\infty}=0$, and the minus sign in the integrand would be a plus sign.

Hard sphere fluid: We start from the expressions for $\mathcal{L}=L_{+}$and $\mathcal{L}^{\epsilon}=-L_{-}$in the notation of hard sphere kinetic theory $[7,15,16,5]$, i.e.

$$
L_{ \pm}=L_{0} \pm \sum_{i<j} T_{ \pm}(i j) ; \quad \bar{L}_{ \pm}=L_{0} \pm \sum_{i<j} \bar{T}_{ \pm}(i j) .
$$

Here $L_{0}=\sum_{i} \mathbf{v}_{i} \cdot \partial / \partial \mathbf{r}_{i}$ is the inertial part. The binary collision operators satisfy the relations $\bar{T}_{ \pm}=\widetilde{T}_{ \pm}^{\epsilon}$ and $T_{ \pm}^{\epsilon}=T_{\mp}$, and their explicit forms can be found in the literature [7]. Then the linear response theory leads to the generalized Green-Kubo formulas (2) for the Navier-Stokes transport coefficients in hard sphere fluids, which reads e.g. for the shear viscosity,

$$
\eta=\eta_{\infty}+(\beta / V) \int_{0}^{\infty} d t\left\langle J_{-x y} e^{t L_{+}} J_{+x y}\right\rangle_{0} .
$$

Here $J_{x y}$ is the total momentum current, obtained for small $k$ from the relation $L_{ \pm} g_{\mathbf{k} \perp} \simeq$ $-i k J_{ \pm x y}=-i k\left(J_{x y}^{k}+J_{ \pm x y}^{v}\right)$, where $(k)$ refers to the kinetic flux, $J_{x y}^{k}=\sum_{i} m v_{i x} v_{i y}$, and $(v)$ to the collisional transfer flux,

$$
\begin{aligned}
J_{ \pm x y}^{v} & = \pm \sum_{i<j} T_{ \pm}(i j) m\left(v_{i y} r_{i x}+v_{j y} r_{j x}\right) \\
& =\sum_{i<j} m \sigma^{d} \int^{(\mp)} d \hat{\sigma}\left(\mathbf{v}_{i j} \cdot \hat{\sigma}\right)^{2} \hat{\sigma}_{x} \hat{\sigma}_{y} \delta\left(\mathbf{r}_{i j}-\sigma \hat{\sigma}\right)
\end{aligned}
$$

The constraint $(\mp)$ on the $\hat{\sigma}$-integration restricts that integral to the pre/post-collision hemisphere where $\mp \mathbf{v}_{i j} \cdot \hat{\sigma} \geq 0$. The explicit expressions for the currents, which are identical to those in Ref.[5], are very different from the ones appearing in the standard form (1), and also very different from those in those in the Langevin fluids. The instantaneous viscosity $\eta_{\infty}$ is proportional to $k^{-2}\left\langle g_{\mathbf{k} \perp} \mid \mathcal{L} g_{\mathbf{k} \perp}\right\rangle$, and is calculated in Ref. [5] as $\eta_{\infty}=\rho \sigma^{2} /\left[d(d+2) t_{E}\right]$, where $\sigma$ is the diameter of the hard spheres, and $t_{E}$ the Enskog mean free time. The same instantaneous contributions can also be identified [15, 17] in the Enskog theory [18] for the hard sphere fluid. Details are presented in [17].

We conclude with a number of comments:

(i) We have derived generalized Green-Kubo formulas, Eq. (2), for non-conservative systems, ranging from microscopic hard sphere fluids with elastic impulsive interactions, to mesoscopic Langevin fluids with weak dissipative and stochastic interactions. Moreover a fundamental commutation relation (4) has been formulated which guarantees that the time correlation functions are stationary, and characterizes those systems for which the transport coefficients 
are given by the generalized form (2). For conservative systems the generalized formulas reduce to the standard Green-Kubo expressions (1).

(ii) The generalized formula (2) does not apply to fluids of inelastic hard spheres, which are models for granular fluids, although infinitesimal generators of the form (14) have been constructed. The reason is that such dissipative systems, either freely cooling or driven by an energy source, do not reach a stationary state described by the thermal distribution $\rho_{0}$.

(iii) From the existence of pseudo-streaming operators for hard sphere fluids an interesting side result can be deduced. It allows us to introduce pseudo-interparticle forces $[4,5], \mathbf{F}_{i j}^{p s}$ and conjugate forces through the relation $\mathcal{L} m \mathbf{v}_{i}=\sum_{j(\neq i)} \mathbf{F}_{i j}^{p s}$, and $\mathcal{L}^{\epsilon} m \mathbf{v}_{i}=\sum_{j(\neq i)} \mathbf{F}_{\epsilon, i j}^{p s}$ respectively. An identity, derived in [5], states that $\left\langle\mathbf{F}_{\epsilon, i j, x}^{p s}(0) \mathbf{F}_{i j, y}^{p s}(t)\right\rangle_{0}=\mathcal{C} \delta_{x y} \delta(t)$, where $\mathcal{C}$ is given explicitly. Comparison with the time correlation function for the Langevin forces suggests that the pseudo-interparticle forces in hard sphere systems can be considered as white noise.

(iv) Another side result of interest, following from (14), is the observation that the frequently cited Green-Kubo formulas for the DPD fluid [19], regarding for the viscosity for instance, $\eta=\eta^{c c}-\eta^{d d}$, are not correct (compare with (13)). Equally incorrect is the standard Green-Kubo formula (1) for the heat conductivity of the random solid, quoted in Ref.[10] as $\lambda=-\lambda^{d d}$ (compare with (12)). It was mentioned there as a possible alternative for the kinetic theory analysis, presented in that paper. Regarding the main interest of this letter, i.e. the generic structure of the Green-Kubo formulas for non-conservative systems, the last comment is only of minor importance.

Acknowledgments: The authors thank J. Piasecki and P. Español for bringing Refs.[4] and [6] respectively to their attention. M.H.E. is supported by Secretaría de Estado de Educación y Universidades (Spain), and R.B. by the Universidad Complutense (Profesores en el Extranjero). This work is financed by the research project FIS2004-271 (Spain).

\section{REFERENCES}

[1] J.P. Hansen and I.R. McDonald, Theory of Simple Liquids (Wiley, New York, 1986), Chap 8.

[2] M.P. Allen and D.J. Tildesley, Computer Simulations in Liquids, (Wiley, New York, 1987).

[3] M.H. Ernst and J.R. Dorfman, J. Stat. Phys. 12, 311 (1975).

[4] L. Bocquet, J. Piasecki, and J.P. Hansen, J. Stat. Phys. 76, 505 (1994), and J. Piasecki, L. Bocquet and J.P. Hansen, Physica A 218, 125 (1995).

[5] J.W. Dufty and M.H. Ernst, Molecular Physics, 102, 2123 (2004), and cond-mat/0401635.

[6] P. Español and F. Vázquez, Phil. Trans. R. Soc., A 360, 383 (2002).

[7] M.H. Ernst, J.R. Dorfman, W.R. Hoegy and J.M.J. van Leeuwen, Physica (Utrecht) 45, 127 (1969).

[8] H. Risken, The Fokker-Planck Equation, (Springer, Berlin, 1996).

[9] M. Ripoll, P. Español, and M.H. Ernst, Int. J. of Mod. Phys. C 9, 1329 (1998).

[10] M. Ripoll and M.H. Ernst, Phys. Rev. E 71, 041104 (2005).

[11] P.J. Hoogerbrugge and J.M.V.A. Koelman, Europhys. Lett. 19, 191 (1995).

[12] P. Español and P. Warren, Europhys. Lett. 30, 191 (1995).

[13] C.A. Marsh, G. Backx and M.H. Ernst, Phys. Rev. E, 56, 1676 (1997).

[14] I. Pagonabarraga, M.H.J. Hagen and D. Frenkel, Europhys. Lett. 42, 377 (1998).

[15] H. van Beijeren and M.H. Ernst, J. Stat. Phys. 21, 125 (1979).

[16] I. de Schepper, M.H. Ernst, and E.G.D. Cohen, J. Stat. Phys. 25, 321 (1981).

[17] M.H. Ernst and R. Brito, Phys. Rev. E, 72, 061102 (2005).

[18] S. Chapman and T. G. Cowling, The Mathematical theory of Non-uniform Gases, (Cambridge Universirty Press, 1970, third edition). 
[19] P. Español, Phys. Rev. E 52, 1734 (1995). 